\title{
Estudo da autodepuração do Rio Jordão, localizado na bacia hidrográfica do Rio Dourados
}

\section{Self-depuration study of Jordão River, located in Dourados River Basin}

\author{
Marcio Ricardo Salla \\ Engenheiro Civil. Professor Adjunto III da Universidade Federal de Uberlândia (UFU) - Uberlândia (MG), Brasil. \\ Carlos Eugênio Pereira \\ Engenheiro Civil. Professor Adjunto II da UFU - Uberlândia (MG), Brasil. \\ José Eduardo Alamy Filho \\ Engenheiro Civil. Professor Adjunto III da UFU - Uberlândia (MG), Brasil. \\ Liliane Magnavaca de Paula \\ Arquiteta. Mestre pela UFU - Uberlândia (MG), Brasil. \\ Aline Martins Pinheiro \\ Química. Mestranda na UFU - Uberlândia (MG), Brasil.
}

\section{Resumo}

O presente artigo avaliou a capacidade de autodepuração do Rio Jordão, considerando as contribuições reais do córrego Brejo Alegre em período de estiagem e os dois cenários que levam em conta as cargas poluidoras estimadas da estação de tratamento de esgoto e a baixa capacidade de diluição do rio para a vazão crítica $Q_{7,10}$, por meio do modelo QUAL-UFMG. A simulação na estiagem apresentou aceitável calibração, tendo em vista a otimização dos coeficientes, evidenciando a prevalência da desoxigenação por demanda carbonácea sobre a nitrificação e a importância da reaeração natural no processo de autodepuração. Apenas o parâmetro 'demanda bioquímica de oxigênio' ( $\left.\mathrm{DBO}_{5}\right)$ não respeitou os limites preconizados pela Resolução do Ministério do Meio Ambiente 357:2005, tendo ficado em toda a extensão do rio fora da regulamentação devido ao recebimento de cargas poluidoras do córrego Brejo Alegre. Quanto às simulações dos cenários, as cargas poluidoras de uma população de 13.300 habitantes para o Cenário 1 e 20.500 para o 2 garantiriam a capacidade de autodepuração, considerando-se uma vazão crítica 3,2 vezes inferior à menor vazão medida no período de estiagem.

Palavras-chave: autodepuração; modelo QUAL-UFMG; Rio Jordão; córrego Brejo Alegre.

\section{Abstract}

The present article assessed the self-depuration capacity of Jordão River, considering the real contributions of Brejo Alegre stream in a drought period and two scenarios regarding the estimated polluting loads of a waste treatment plant and the low dilution capacity of the river for the $Q_{7,10}$ critical flow by using the QUAL-UFMG model. The simulation for the drought period presented acceptable calibration by adjusting coefficients, evidencing the deoxygenation by carbon demand over nitrification and the importance of natural re-aeration in the self-depuration process. Only the biochemical oxygen demand $\left(\mathrm{BOD}_{5}\right)$ parameter did not respect the limits recommended by the CONAMA Resolution 357:2005, in which all the river extension was outside the regulations due to the pollution loads received from Brejo Alegre stream. For the simulated scenarios, polluting loads of a population of 13.300 people for scenario 1 and 20.500 for 2 guaranteed the self-depuration capacity, considering a critical flow 3.2 times lower than the lowest flow measured in the drought period.

Keywords: self-depuration; QUAL-UFMG model; Jordão River; Brejo Alegre stream. 


\section{Introdução}

As ações humanas sobre o meio ambiente, sem certa preocupação com os impactos ambientais, demonstraram prejuízos atuais e fizeram refletir quanto à necessidade de agir, a fim de garantir um meio ambiente sustentável. Existem inúmeros exemplos de degradações e suas consequências ao meio ambiente e ao homem, tais como contaminações e assoreamentos dos cursos de águas naturais, além de enchentes que causam prejuízos principalmente nos centros urbanos.

A literatura da área e os meios de comunicações apresentam uma crescente deterioração ambiental do planeta e, neste cenário, a escassez de água com boa qualidade surge como um dos grandes e importantes problemas que a humanidade precisa enfrentar neste início do século 21, agravada nas bacias hidrográficas com maiores índices de urbanização, causada pelo rápido crescimento da demanda de água e pela poluição dos cursos de água advinda do lançamento de águas residuárias sem nenhum tipo de tratamento.

"De maneira geral, pode-se dizer que a qualidade de uma determinada água é função do uso e da ocupação do solo na bacia hidrográfica" (VON SPERLING, 2007). Em condições de carência de recursos financeiros, presente na maior parte dos municípios brasileiros, a autodepuração, isto é, a capacidade dos corpos de água restabelecerem o equilíbrio do meio aquático por meio de mecanismos naturais após as alterações provocadas pelos despejos dos efluentes, surge como opção quando utilizada segundo os critérios técnicos.

O desenvolvimento sustentável e o uso racional de água requerem que haja uma relação entre a quantidade e a qualidade da água. De acordo com Paredes, Andreu e Solera (2010), existem muitos trabalhos científicos sobre gestão de bacia hidrográfica nos quais são desconsiderados os aspectos de qualidade da água. Devido a esta situação, diversos pesquisadores estão empenhados em relacionar a qualidade da água dentro de um sistema de suporte de decisão no gerenciamento de água em escala de bacia hidrográfica, tais como Arnold e Orlob (1989), Strzepek, García e Over (1989) e Paredes e Lund (2006).

A utilização de um modelo matemático de qualidade da água permite avaliar os impactos do lançamento das cargas poluidoras e analisar cenários de intervenção e medidas de controle ambiental. Na história, esses modelos vêm sendo utilizados desde o desenvolvimento do modelo clássico de Streeter e Phelps, em 1925, criado para o Rio Ohio, tornando-se um marco na história da engenharia sanitária e ambiental. Aumentando-se o grau de complexidade e o número de variáveis modeladas, outros modelos foram propostos, tais como QUAL2E (CHAPRA, 1997) e QUAL2K (CHAPRA; PELLETIER, 2006) e sua modificações, AQUASIM (REICHERT, 1998), GESCAL (PAREDES; ANDREU; SOLERA, 2010), entre outros.

Von Sperling (2007) criou um modelo simplificador das versões QUAL2E e QUAL2K, desenvolvidos inicialmente pela US Environmental Protection Agency (USEPA), chamado QUAL-UFMG, no qual não se leva em consideração a influência de algas e suas inter-relações, visto que são importantes apenas em ambientes lênticos. Em função da facilidade de manuseio, apresenta satisfatória aceitação, sendo utilizado por Pereira et al. (2011), Da Silva et al. (2011), Amorim Junior, Lima e Vieira (2011), Guedes (2009) e Ide e Ribeiro (2009).

O modelo permite simular o comportamento de diversos indicadores e parâmetros de qualidade da água, como, por exemplo: demanda bioquímica de oxigênio (DBO), oxigênio dissolvido (OD), nitrogênio total e frações, fósforo total e frações e coliformes termotolerantes (VON SPERLING, 2007).

O modelo incorpora os fenômenos, mecanismos e processos interagentes no balanço do oxigênio dissolvido, modelando-os em condições de anaerobiose, considerando a sedimentação da matéria orgânica, o consumo de OD pela nitrificação (oxidação da amônia a nitrito e, deste, a nitrato) e as cargas difusas internas sem vazão (demanda do sedimento, fotossíntese e respiração) e externas. Algumas importantes simplificações assumidas pelo modelo podem ser consultadas em De Paula (2011), Pereira et al. (2011) e Da Silva et al. (2011).

O município de Araguari, localizado no Estado de Minas Gerais, com crescente urbanização associada à falta de planejamento, gera impactos pontuais e difusos no córrego Brejo Alegre, afluente do Rio Jordão. Com isso, os objetivos foram: avaliar a capacidade de autodepuração do Rio Jordão, considerando as contribuições reais do córrego Brejo Alegre no período de estiagem, e simular cenários de lançamentos de cargas poluidoras efluentes de estação de tratamento de esgoto (ETE), lançadas diretamente na confluência do córrego com o Rio Jordão para período crítico (vazão $Q_{7,10}$ ). Os resultados poderão contribuir ao melhor planejamento no desenvolvimento urbano do município de Araguari, objetivando sempre preservar a vida aquática e a capacidade de decomposição da matéria orgânica e dos nutrientes transportados até o curso de água.

\section{Metodologia}

\section{Local de estudo}

A área de estudo deste artigo foi um trecho do Rio Jordão, pertencente à Bacia Hidrográfica do Rio Dourados - UPGRH PN1. A sub-bacia hidrográfica do Rio Jordão localiza-se a Oeste do Estado de Minas Gerais, entre as coordenadas 18²5’41" e 18 50'51" de la-

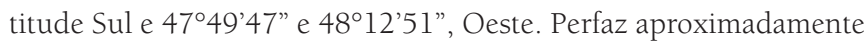
$95 \mathrm{~km}$, desde a nascente até a sua foz no Rio Paranaíba. O Rio Paranaíba é um dos afluentes do Rio Grande, o qual integra a Bacia Transnacional do Rio Paraná.

A sub-bacia do Rio Jordão tem como principal afluente o córrego Brejo Alegre, que se encontra poluído. Foram escolhidos cinco postos de monitoramento fluviométrico e de qualidade da água, 
sendo quatro posicionados ao longo do trecho do Rio Jordão estudado, desde a confluência com o córrego Brejo Alegre até a foz no Rio Paranaíba totalizando aproximadamente $43,5 \mathrm{~km}$, e um posto no próprio córrego Brejo Alegre. A Figura 1 ilustra a bacia estudada com identificações dos postos de monitoramento.

\section{Determinação da vazão média}

No Brasil, devido ao alto custo de implantação e manutenção, existem deficiências de redes hidrométricas e, consequentemente, de dados históricos e, na maioria das vezes, as redes existentes dificilmente cobrem os locais de interesse necessários para o planejamento hídrico de uma região.

Neste artigo, as vazões médias em cada posto fluviométrico (Figura 1) foram obtidas por meio dos trabalhos de campo, multiplicando a velocidade média pela área da seção transversal do curso de água. A partir da seção transversal real levantada com auxílio de um teodolito, utilizando divisões de $1 \mathrm{em} 1 \mathrm{~m}$ em cabo de aço esticado na seção transversal, fixaram-se algumas verticais fictícias ao longo desta seção (formando-se subáreas transversais, como visto na Figura 2). Nesta, foram medidas as velocidades pontuais em pontos específicos de tais verticais pelo molinete fluviométrico, cuja quantidade de pontos é função da profundidade média de cada subárea. A vazão total é a soma das vazões das subáreas, calculadas pela multiplicação da velocidade média por sua correspondente subárea. A Figura 2 ilustra os pontos de fixação do molinete fluviométrico para determinar a vazão média no posto A. Uma descrição mais detalhada da metodologia pode ser consultada em De Paula (2011)

As vazões dos afluentes menores e difusa incremental foram estimadas a partir da específica $\left(\mathrm{m}^{3} \cdot \mathrm{s}^{-1} \cdot \mathrm{km}^{-2}\right)$, conforme descrita na Equação 2.

\section{Determinação da vazão crítica}

Para o planejamento e gerenciamento de uma bacia hidrográfica, a fim de determinar a capacidade mínima de diluição de um curso de água, consideraram-se as condições críticas e ocorrem exatamente no período de vazão mínima. Tal vazão deve ser estimada a partir de dados fluviométricos históricos do curso de água, adotando-se usualmente a mínima para um tempo de recorrência de dez anos e mínima de sete dias $\left(Q_{7,10}\right)$.

Mediante a falta de dados históricos fluviométricos na bacia hidrográfica do Rio Dourados, mais especificamente na sub-bacia do Rio Jordão, ficou inviável a determinação da vazão crítica $Q_{7,10}$. Com
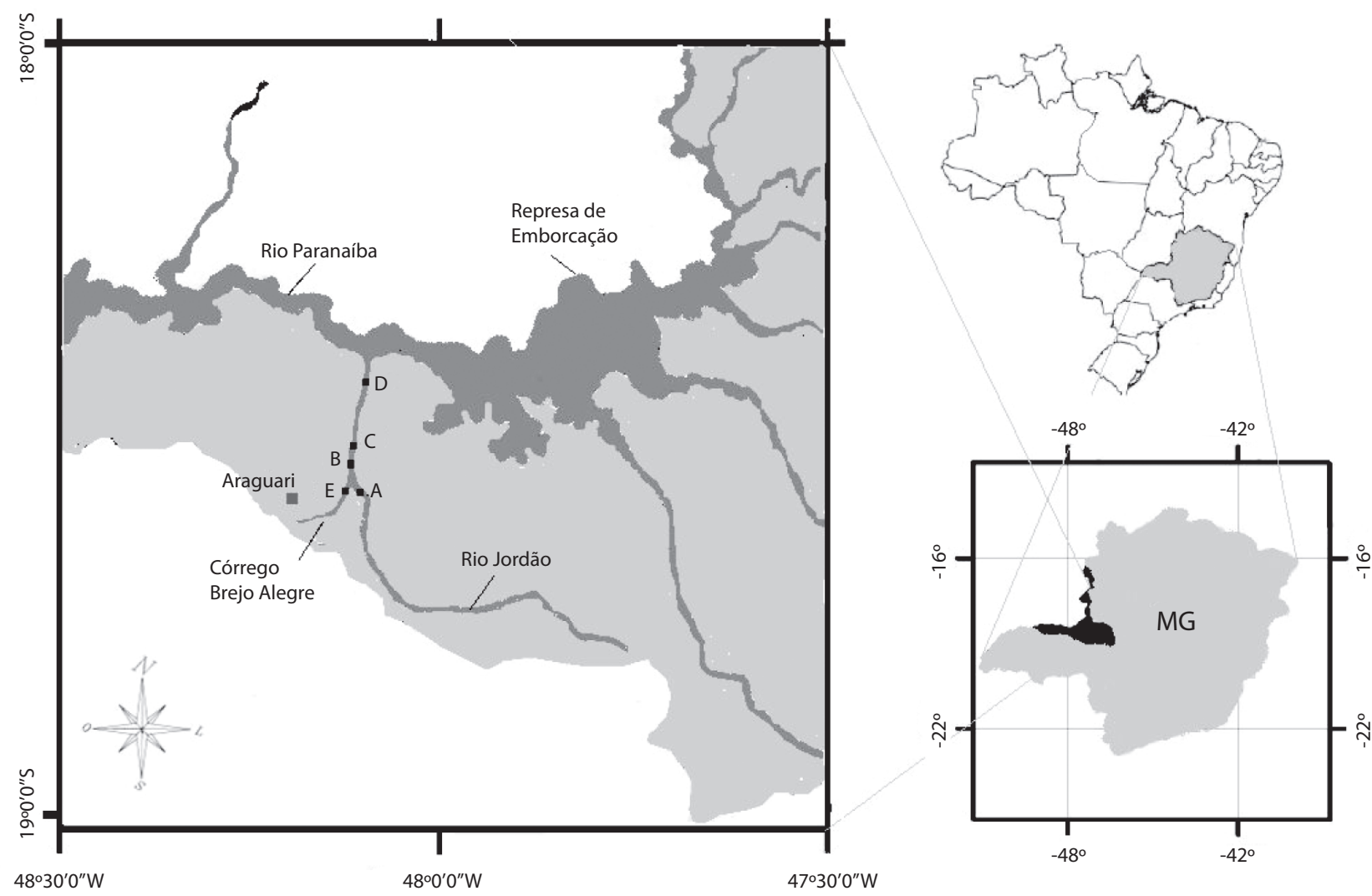

Figura 1 - Identificação dos postos de monitoramento desde o córrego Brejo Alegre (afluente do Rio Jordão) até a confluência do Rio Jordão com o Paranaíba. 
isto, a mesma foi estimada com a utilização de uma ferramenta de gestão hidrológica para o Estado de Minas Gerais, chamada Hidrotec (UNIVERSIDADE FEDERAL DE VIÇOSA, 2009). Essa ferramenta permite estimar dados hidrológicos para as bacias hidrográficas situadas no Estado de Minas Gerais, e para a sub-bacia hidrográfica do Rio Jordão forneceu a equação ajustada da vazão crítica em função da área da bacia contribuinte, sendo $Q_{7,10}=0,0038 \cdot A^{0,9475}$, na qual $Q_{7,10}$ é a vazão crítica em sete dias consecutivos, considerando dez anos de período de retorno $\left(\mathrm{m}^{3} \cdot \mathrm{s}^{-1}\right)$ correspondente ao posto fluviométrico com área de drenagem $A\left(\mathrm{~km}^{2}\right)$. Neste artigo, estimou-se a vazão crítica nos postos A e E (córrego Brejo alegre) e nos 18 afluentes existentes até a confluência com o Rio Paranaíba, por meio de uma ferramenta chamada Geographic Information System (GIS), que possibilita o traçado perimetral diretamente na plataforma Google Earth com a obtenção simultânea da área de contribuição.

\section{Dados de entrada para a modelagem}

As entradas do modelo de simulação da qualidade da água incluem: dados do rio a montante do ponto inicial (posto A), dados físicos do rio, coeficientes de decaimento e aumento da concentração de compostos, dados pontuais e distribuídos para os afluentes e esgotos e padrões de qualidade da água especificados pela Resolução do Ministério do Meio Ambiente (Conama) 357:2005, respeitando-se a classe 2 do Rio Jordão:

- dados do rio a montante (cabeceira), no posto A: vazão $\left(\mathrm{m}^{3} \cdot \mathrm{s}^{-1}\right)$, OD (mg. $\left.\mathrm{L}^{-1}\right), \mathrm{DBO}_{5}\left(\mathrm{mg} \cdot \mathrm{L}^{-1}\right)$, nitrogênio orgânico (mg. $\left.\mathrm{L}^{-1}\right)$, amônia (mg. $\left.\mathrm{L}^{-1}\right)$, nitrito (mg. $\left.\mathrm{L}^{-1}\right)$, nitrato (mg. $\left.\mathrm{L}^{-1}\right)$, fósforo (mg. $\left.\mathrm{L}^{-1}\right)$, E-coli (NMP. $\left.100 \mathrm{~mL}^{-1}\right)$ e temperatura $\left({ }^{\circ} \mathrm{C}\right)$;

- dados físicos do rio: distância do trecho $(\mathrm{km})$, altitude $(\mathrm{m})$, velocidade $v\left(\mathrm{~m} . \mathrm{s}^{-1}\right)$ e profundidade $h(\mathrm{~m})$. O trecho foi dividido em segmentos de $100 \mathrm{~m}$, que viabilizou uma melhor integração dos parâmetros simulados. O modelo original utiliza a altitude e a temperatura do ponto de mistura (confluência do córrego Brejo Alegre com o Rio Jordão) para determinar a concentração de saturação de $\mathrm{OD}$ em qualquer ponto. As concentrações de saturação de OD foram estimadas para cada segmento de $100 \mathrm{~m}$, considerando a declividade constante entre os trechos A-E, E-B, B-C e C-D e a temperatura para cada um igual à temperatura do ponto a montante de seu respectivo trecho. Diante da hipótese de escoamento unidimensional, uniforme e permanente, adotada por este modelo de qualidade da água e conhecidas as áreas das seções transversais dos postos fluviométricos (Figura 1), as relações hidráulicas entre velocidade média do escoamento $v$ (m.s $\left.{ }^{1}\right)$, profundidade média do escoamento $h(\mathrm{~m})$ e vazão $\mathcal{Q}\left(\mathrm{m}^{3} \cdot \mathrm{s}^{-1}\right)$, para cada segmento de $100 \mathrm{~m}$ em todos os trechos simulados, foram obtidas por meio da equação da continuidade. Para isso, por meio de resolução numérica por iteração, a profundidade média $h$ foi obtida diante da igualdade da função do escoamento (dependente da vazão $Q$, declividade de fundo $I\left(\mathrm{~m} . \mathrm{m}^{-1}\right)$ e rugosidade das paredes $\eta\left(\mathrm{m}^{-1 / 3}\right.$.s $)$ ) com a função geométrica da seção transversal (seção trapezoidal é dependente da largura da base $b(\mathrm{~m})$, inclinação dos taludes $\theta$ e profundidade média $h(\mathrm{~m}))$, cuja formulação matemática está ilustrada na Equação 1. De acordo com a Equação 1, neste estudo, considerou-se mesma inclinação de talude para as margens do curso de água.

$(Q \cdot \eta) / I^{1 / 2}=\left[\left(b \cdot h+h^{2} \cdot \operatorname{cotg} \theta\right)^{5 / 3}\right] /\left[b+2 \cdot\left(h^{2} \cdot\left(\operatorname{cotg} \theta^{2}+1\right)^{1 / 2}\right]^{2 / 3}\right.$

- coeficientes considerados: desoxigenação $K_{1}\left(\right.$ dia $\left.^{-1}\right)$, decomposição $K_{d}\left(\right.$ dia $\left.^{-1}\right)$, sedimentação $K_{s}\left(\right.$ dia $\left.^{-1}\right)$, reaeração $K_{2}\left(\right.$ dia $\left.^{-1}\right)$, sedimentação do nitrogênio orgânico $K_{\mathrm{so}}\left(\right.$ dia $\left.^{-1}\right)$, conversão do nitrogênio orgânico em amoniacal $K_{\text {oa }}\left(\right.$ dia $\left.^{-1}\right)$, conversão do nitrogênio amoniacal em nitrito $K_{\mathrm{an}}\left(\right.$ dia $\left.^{-1}\right)$, conversão do nitrito em nitrato $K_{\mathrm{nn}}\left(\mathrm{dia}^{-1}\right)$, liberação do nitrogênio amoniacal pelo sedimento de fundo $S_{\text {namon }}\left(\mathrm{g} \cdot \mathrm{m}^{-2} \cdot \mathrm{dia}^{-1}\right), \mathrm{O}_{2}$ equivalente na conversão do nitrogênio amoniacal em nitrito $\mathrm{O}_{2 \mathrm{Namon}}\left(\mathrm{mgO}_{2} \cdot \mathrm{mgN}_{\text {amon oxid }}{ }^{-1}\right), \mathrm{O}_{2}$ equivalente na conversão do nitrito em nitrato $\mathrm{O}_{2 \mathrm{Nnitri}}\left(\mathrm{mgO}_{2} \cdot \mathrm{mgN}_{\text {nitrito oxid }}{ }^{-1}\right)$, inibição da nitrificação por baixo OD $K_{\text {nitr }}\left(\right.$ dia $\left.^{-1}\right)$, sedimentação do fósforo orgânico $K_{\text {spo }}\left(\right.$ dia $\left.^{-1}\right)$, conversão do fósforo orgânico em inorgânico $K_{\mathrm{oi}}\left(\right.$ dia $\left.^{-1}\right)$, liberação do fósforo inorgânico pelo sedimento de fundo $S_{\text {pinorg }}\left(\mathrm{g} \cdot \mathrm{m}^{-2} \cdot \mathrm{dia}^{-1}\right)$ e decaimento de coliforme $K_{\mathrm{b}}\left(\mathrm{dia}^{-1}\right)$. Todavia, não foram consideradas neste artigo: taxa de produção de $\mathrm{O}_{2}$ por fotossíntese $P^{\prime}\left(\mathrm{g} \cdot \mathrm{m}^{-2} \cdot \mathrm{dia}^{-1}\right)$, taxa de consumo de $\mathrm{O}_{2}$ por respiração $R^{\prime}\left(\mathrm{g} \cdot \mathrm{m}^{-2} \cdot \mathrm{dia}^{-1}\right)$, taxa de consumo de $\mathrm{O}_{2}$ por demanda do sedimento $S d^{\prime}\left(\mathrm{g} \cdot \mathrm{m}^{-2} \cdot \mathrm{dia}^{-1}\right)$ e carga linear distribuída de $\mathrm{DBO}_{5}$ ao longo do rio Lrd' $\left(\mathrm{gDBO}_{5} \cdot \mathrm{m}^{-1} \cdot \mathrm{dia}^{-1}\right)$;

- dados pontuais dos afluentes: vazão, $\mathrm{DBO}_{5}, \mathrm{OD}$, nitrogênio orgânico, nitrogênio amoniacal, nitrito, nitrato, fósforo e coliforme.

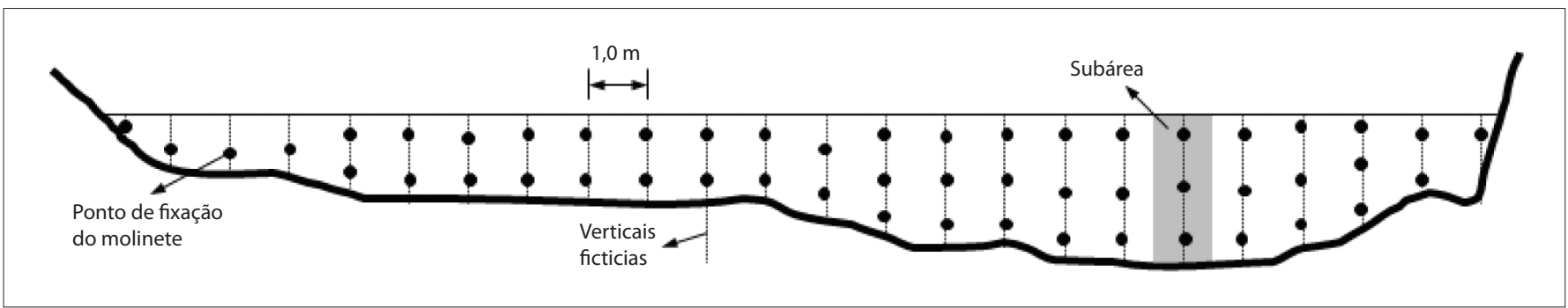

Figura 2 - Pontos de fixação do molinete fluviométrico na seção transversal do posto A. 
O modelo aceita como dados pontuais esgoto, afluentes e subtração de vazão no caso de captação de água; e como distribuídos, contribuição incremental de vazão e esgotos;

- padrões de qualidade especificados pela legislação ambiental, baseados na Resolução Conama 357:2005 para a Classe 2 no Rio Jordão.

\section{Simulação no período de estiagem}

Como dados de entrada pontuais, consideraram-se o posto A, o córrego Brejo Alegre e outros 18 afluentes menores, sendo a qualidade da água analisada no posto A, no córrego Brejo Alegre e no Afluente 1. Não foi possível realizar análises de qualidade nos afluentes 2 a 18, devido às dificuldades logísticas, principalmente com relação aos acessos aos afluentes. A estimativa da qualidade de tais afluentes similar aos parâmetros físicos, químicos e biológicos encontrados no Afluente 1 está embasada na similaridade de uso do solo nessas sub-bacias.

Diante da limitação deste estudo com relação à falta do monitoramento de qualidade da água na maioria dos afluentes menores, realizou-se uma análise de sensibilidade da variação de qualidade da água ao longo do trecho do rio estudado, diante de diversas mudanças na qualidade dos Afluentes 2 a 18 e para a descarga difusa (contempla os afluentes menores com descarga direta no rio). Nesta análise da sensibilidade consideraram-se, como situação mais crítica, mudanças percentuais iguais no valor quantitativo para todos os parâmetros e simultâneas para os 17 afluentes e descarga difusa. Tendo-se como referência os parâmetros de qualidade do Afluente 1, avaliou-se a sensibilidade aumentando-se os valores dos parâmetros de 20 a 100\% em intervalos de 20\%. Para o parâmetro OD, analisou-se a sensibilidade para os valores de 5,2 mg. $\mathrm{L}^{-1}(80 \%$ do OD no Afluente 1), 3,9 mg. $\mathrm{L}^{-1}(60 \%$ do OD no Afluente 1) e 2,6 mg. $\mathrm{L}^{-1}$ (40\% do OD no Afluente 1).

A vazão pontual para cada um dos 18 afluentes $\mathcal{Q}_{\mathrm{i}}$ foi estimada a partir da descarga específica $\left(\mathrm{m}^{3} \cdot \mathrm{s}^{-1} \cdot \mathrm{km}^{-2}\right)$, levando-se em consideração medidas realizadas no curso de água em um posto a montante e outro a jusante (postos B, C e D), além da quantidade de afluentes neste intervalo, conforme Equação 2:

$Q_{\mathrm{i}}=\left[\left(Q_{\text {rio Jordāo a jusante }}-Q_{\text {rio Jordâo a montante }}\right) / \sum A_{n}\right] . A_{i}$

onde, $A_{\mathrm{n}}$ é a área total entre dois postos de monitoramento sequenciais e $A_{i}$ é aquela de contribuição do Afluente i. Já a vazão difusa para cada incremento de $0,1 \mathrm{~km}\left(Q_{\text {difusa }}\right)$ foi estimada por meio da Equação 3:

$Q_{\text {difusa }}=c t e \cdot\left[\left(\left(Q_{\text {rio Jordào a jusante }}-Q_{\text {rio Jordäo a montante }}\right) / \sum A_{n}\right) \cdot A_{\text {difusa }}\right]$

onde: $A_{\text {difusa }}$ é a área difusa e cte é=2,298.10-3 (incremento de 0,1 km dividido pelo trecho total simulado de $43,5 \mathrm{~km}$ ).
Os dados de entrada dos postos A, B, C, D e E foram medidos em campo e em laboratório, cujos resultados estão ilustrados na Tabela 2. Os dados dos postos B, C e D, além de possibilitar a estimativa de vazão nos afluentes e difusa incremental, foram utilizados nas calibrações dos coeficientes. A Figura 3 ilustra o diagrama unifilar, com todos os afluentes e postos fluviométricos e de monitoramento pertencentes ao trecho. Entre os meses de abril até julho de 2011, houve quatro sequências de deslocamento aos locais de estudo para determinações de vazão, análises de alguns parâmetros de qualidade no local (temperatura, pH e oxigênio dissolvido), além da coleta de amostras para as análises laboratoriais.

\section{Simulações dos cenários}

Foram simulados os Cenários 1 e 2, considerando, no 1, que o esgoto sanitário bruto da área urbana do município de Araguari (MG) recebe o tratamento secundário formado por lagoa anaeróbia, seguida por facultativa (sistema australiano) antes de ser lançado na confluência do córrego Brejo Alegre com o Rio Jordão. No Cenário 2, levou-se em conta que o esgoto sanitário bruto recebe o tratamento secundário formado por lodo ativado convencional.

Nas simulações dos cenários foram considerados os mesmos parâmetros e coeficientes do período de estiagem, além de estimar a vazão crítica $Q_{7,10}$ para o Rio Jordão, córrego Brejo Alegre e os 18 afluentes em função da área de contribuição, conforme descrito anteriormente.

Os dados de entrada do efluente doméstico nos Cenários 1 e 2, referentes às cargas poluidoras e eficiência de remoção para cada parâmetro, além dos limites preconizados na Resolução Conama 430:2011, são apresentados na Tabela 1 .

A vazão de esgoto sanitário $\mathcal{Q}_{\text {esgoto }}$ foi calculada pela equação da vazão de distribuição, multiplicada pelo coeficiente de retorno, que, de acordo com a NBR da ABNT 9649 (1986) e 14486 (2000), é fixado em 0,80 em situações em que não existirem dados locais comprovados oriundos de pesquisas.

A carga per capita para a DBO (Tabela 1) foi adotada conforme a recomendação da ABNT NBR 12209 (1992) na ausência de trabalho experimental no local de interesse. Já as cargas poluidoras dos outros parâmetros e as eficiências de remoções (limites mínimos) foram estimadas considerando a elevada quantidade de resultados experimentais relatados por diversos autores, tais como Von Sperling (2007), Tchobanoglous, Burton e Stensel (2003) e Alem Sobrinho (2001), válido para os processos de tratamento de efluentes assumidos nos cenários. Desta forma, observa-se que, para os Cenários 1 e 2, os dados de entrada dos parâmetros dependem da população $P$ do município. Com isto, essas simulações tiveram o propósito de verificar qual a máxima população $P$ que os sistemas de tratamento suportariam para que, após lançado diretamente no Rio Jordão, a autodepuração natural do curso de água conseguisse adequar os parâmetros aos padrões da Resolução Conama 357 (2005), para a Classe 2. Vale ressaltar que a população total do município estimada pelo Instituto 
Brasileiro de Geografia e Estatística (IBGE), em 2010, era de 109.800 habitantes e que o efluente doméstico foi lançado diretamente no córrego Brejo Alegre sem nenhum tipo de tratamento prévio (BRASIL, 2010).

\section{Resultados}

\section{Simulações hidráulicas}

Conforme mencionado, no processo iterativo utilizado nas simulações hidráulicas, a partir das seções transversais conhecidas, obteve-se, para cada segmento de $100 \mathrm{~m}$ dentro do trecho de rio estudado, a profundidade média ótima que iguala a função do escoamento com a geométrica da seção transversal. Para as simulações no período de estiagem, a velocidade média manteve-se entre 0,78 e 0,92 m.s.1, a profundidade média entre 0,32 e 0,66 m e a largura da superfície líquida entre 15,25 e 22,18 m.

\section{Dados quantitativos e qualitativos}

A Tabela 2 ilustra os resultados dos parâmetros medidos e estimados no período de estiagem e os resultados dos dados estimados para simulação dos cenários (vazão $Q_{710}$ ).
A análise da sensibilidade na qualidade de água devido às variações dos parâmetros nos Afluentes 2 a 18 e na descarga difusa foi realizada utilizando-se, como dados comparativos, os resultados estimados de qualidade da água ao longo do trecho de rio após a calibração (Figura 4). Constatou-se o aumento de até $10,4 \%$ para $\mathrm{DBO}_{5}, 4,6 \%$ para nitrogênio total, $8,8 \%$ para fósforo total e 1,5\% para E. coli. Com relação ao OD, obteve-se a diminuição de até 4,3, 6,8 e 9,4\% para 80, 60 e 40\% da concentração de OD no Afluente 1, respectivamente. Esta análise de sensibilidade enfatiza ainda mais a importância do frequente monitoramento da quantidade e da qualidade da água em cursos de água como subsídio para adequada calibração de modelo.

\section{Simulação para o período de estiagem}

A capacidade de autodepuração do Rio Jordão, considerando a contribuição real do córrego Brejo Alegre em período de estiagem, foi analisada com o modelo QUAL-UFMG, tendo como entrada os dados quantitativos e qualitativos (Tabela 2). A Figura 4 apresenta a evolução dos parâmetros em função da distância no trecho do Rio Jordão estudado e os coeficientes utilizados na simulação. Foram calibrados os coeficientes $K_{1}, K_{\mathrm{d}}, K_{\mathrm{s}}, K_{\mathrm{spo}}, K_{\mathrm{oi}}, S p_{\text {inorg }}, K_{\mathrm{b}}, K_{\mathrm{so}}, K_{\mathrm{oa}}, K_{\text {an }}, K_{\text {nn }}, S_{\text {namon }}$ $k_{\text {nitr }}, O_{2 \text { Namon }}$ e $O_{2 \text { Nnitri }}$, utilizando a ferramenta de otimização não linear

Tabela 2 - Dados quantitativos e qualitativos obtidos nos postos monitorados e estimados.

\begin{tabular}{|c|c|c|c|c|c|c|c|}
\hline \multicolumn{8}{|c|}{ Período de estiagem } \\
\hline \multirow{2}{*}{ Parâmetro } & \multirow{2}{*}{ Unidade } & A & B & C & D & $\mathrm{E}$ & Afluentes $^{c}$ \\
\hline & & \multicolumn{5}{|c|}{ Medidos } & Estimados \\
\hline \multirow{2}{*}{$Q_{\text {estiagem }}$} & \multirow{2}{*}{$\mathrm{m}^{3} \cdot \mathrm{s}^{-1}$} & $5,7 \pm 0,2^{\mathrm{a}}$ & $6,7 \pm 0,3^{a}$ & $7,4 \pm 0,4^{a}$ & $8,2 \pm 0,3^{a}$ & $0,39 \pm 0,03^{a}$ & $0,11 \pm 0,12^{a}$ \\
\hline & & $5,4-5,9^{b}$ & $6,3-7,0^{\mathrm{b}}$ & $6,9-7,8^{b}$ & $7,9-8,5^{b}$ & $0,35-0,42^{\mathrm{b}}$ & $0,01-0,35^{b}$ \\
\hline \multirow{2}{*}{ OD } & \multirow{2}{*}{$\mathrm{mg} \cdot \mathrm{L}^{-1}$} & $6,9 \pm 0,7^{a}$ & $6,1 \pm 0,4^{a}$ & $6,3 \pm 0,6^{a}$ & $6,5 \pm 0,6^{a}$ & $5,2 \pm 0,4^{\mathrm{a}}$ & \multirow{2}{*}{6,5} \\
\hline & & $6,2-7,8^{\mathrm{b}}$ & $5,7-6,5^{\mathrm{b}}$ & $5,6-7,0^{\mathrm{b}}$ & $5,8-7,1^{\mathrm{b}}$ & $4,7-5,6^{b}$ & \\
\hline \multirow{2}{*}{ Temperatura } & \multirow{2}{*}{$\stackrel{\circ}{ } \mathrm{C}$} & $18,8 \pm 2,1^{a}$ & $18,7 \pm 2,0^{a}$ & $19,0 \pm 1,3^{a}$ & $19,1 \pm 1,1^{\mathrm{a}}$ & $18,6 \pm 0,8^{a}$ & \multirow{2}{*}{20,0} \\
\hline & & $16,3-21,2^{b}$ & $16,4-21,0^{\mathrm{b}}$ & $17,9-20,5^{b}$ & $18,0-20,4^{b}$ & $18,0-19,5^{b}$ & \\
\hline \multirow{2}{*}{$\mathrm{DBO}_{5}$} & & $7,4 \pm 0,6^{\mathrm{a}}$ & $6.5 \pm 0.4^{\mathrm{a}}$ & $6.1 \pm 1.3^{\mathrm{a}}$ & $6.6 \pm 0.8^{a}$ & $21,4 \pm 4,3^{a}$ & \multirow{2}{*}{2,0} \\
\hline & & $6,7-8,1^{b}$ & $6,1-6.9^{b}$ & $4.9-7,5^{b}$ & $5.8-7.3^{b}$ & $15,3-25,0^{b}$ & \\
\hline \multirow{2}{*}{$\mathrm{N}_{\text {Orgânico }}$} & & $0,3 \pm 0,2^{a}$ & $0,6 \pm 0,4^{a}$ & $0,5 \pm 0,2^{\mathrm{a}}$ & $0,4 \pm 0,2^{\mathrm{a}}$ & $1,2 \pm 0,6^{\mathrm{a}}$ & \multirow{2}{*}{0,2} \\
\hline & & $0,1-0,6^{b}$ & $0,2-0,9^{b}$ & $0,2-0,7^{\mathrm{b}}$ & $0,3-0,7^{b}$ & $0,7-2,0^{b}$ & \\
\hline \multirow{2}{*}{ Amônia } & & $0,7 \pm 0,7^{a}$ & $1,8 \pm 1,7^{a}$ & $1,3 \pm 1,1^{a}$ & $1,1 \pm 1,3^{\mathrm{a}}$ & $6,6 \pm 6,5^{\mathrm{a}}$ & \multirow{2}{*}{0,2} \\
\hline & $\mathrm{mgll}^{-1}$ & $0,3-1,8^{b}$ & $0,3-3,9^{b}$ & $0,5-2,8^{b}$ & $0,4-3,0^{b}$ & $1,3-15,4^{b}$ & \\
\hline \multirow{2}{*}{ Nitrito } & $\mathrm{mg} \cdot \mathrm{L}^{-1}$ & $0,03 \pm 0,04^{a}$ & $0,12 \pm 0,10^{a}$ & $0,10 \pm 0,07^{a}$ & $0,11 \pm 0,08^{a}$ & $0,76 \pm 0,49^{a}$ & \multirow{2}{*}{0,0} \\
\hline & & $0,01-0,09^{b}$ & $0,05-0,27^{b}$ & $0,05-0,20^{b}$ & $0,06-0,23^{b}$ & $0,40-1,48^{b}$ & \\
\hline \multirow{2}{*}{ Nitrato } & & $0,72 \pm 0,58^{a}$ & $0,87 \pm 0,31^{a}$ & $1,20 \pm 0,21^{a}$ & $1,32 \pm 0,19^{a}$ & $4,05 \pm 2,59^{a}$ & \multirow{2}{*}{0,0} \\
\hline & & $0,09-1,26^{b}$ & $0,58-1,15^{b}$ & $0,91-1,41^{b}$ & $1,22-1,61^{b}$ & $2,40-7,91^{b}$ & \\
\hline \multirow{2}{*}{$\mathrm{P}_{\text {Total }}$} & & $0,03 \pm 0,03^{a}$ & $0,09 \pm 0,07^{a}$ & $0,09 \pm 0,08^{a}$ & $0,10 \pm 0,10^{\mathrm{a}}$ & $0,52 \pm 0,59^{\mathrm{a}}$ & \multirow{2}{*}{0,01} \\
\hline & & $0,00-0,06^{b}$ & $0,05-0,20^{b}$ & $0,04-0,20^{b}$ & $0,04-0,24^{b}$ & $0,18-1,40^{b}$ & \\
\hline \multirow{2}{*}{ E-coli } & \multirow{2}{*}{ NMP.100 $\mathrm{mL}^{-1}$} & $18,5 \pm 22,7^{a}$ & $68,3 \pm 69,3^{a}$ & $55,8 \pm 58,4^{a}$ & $40,3 \pm 42,1^{a}$ & $85,0 \pm 58,4^{a}$ & \multirow{2}{*}{$1,1.10^{\circ}$} \\
\hline & & $0-50^{\mathrm{b}}$ & $0-150^{b}$ & $0-120^{\mathrm{b}}$ & $0-80^{\mathrm{b}}$ & $9,2-150^{b}$ & \\
\hline \multicolumn{8}{|l|}{ Cenários $^{d}$} \\
\hline \multirow{2}{*}{ Parâmetro } & \multirow{2}{*}{ Unidade } & A & B & C & D & E & Afluentes \\
\hline & & \multicolumn{6}{|c|}{ Estimados } \\
\hline & $\mathrm{m}^{3} \mathrm{~s}^{-1}$ & 177 & - & - & - & 011 & $0,04 \pm 0,04^{a}$ \\
\hline$Q_{7,10}$ & $\mathrm{~m} \cdot \mathrm{s}$ & $1,7 /$ & - & - & - & 0,11 & $0,004-0,125^{b}$ \\
\hline
\end{tabular}

amédia \pm desvio padrão; bmínimo - máximo; 'dados de qualidade da água nos afluentes 2 a 18 mais descarga difusa, considerados similares aos dados analisados no afluente 1; 'os dados de qualidade da água para o Rio Jordão e os afluentes foram considerados similares ao período de estiagem. 


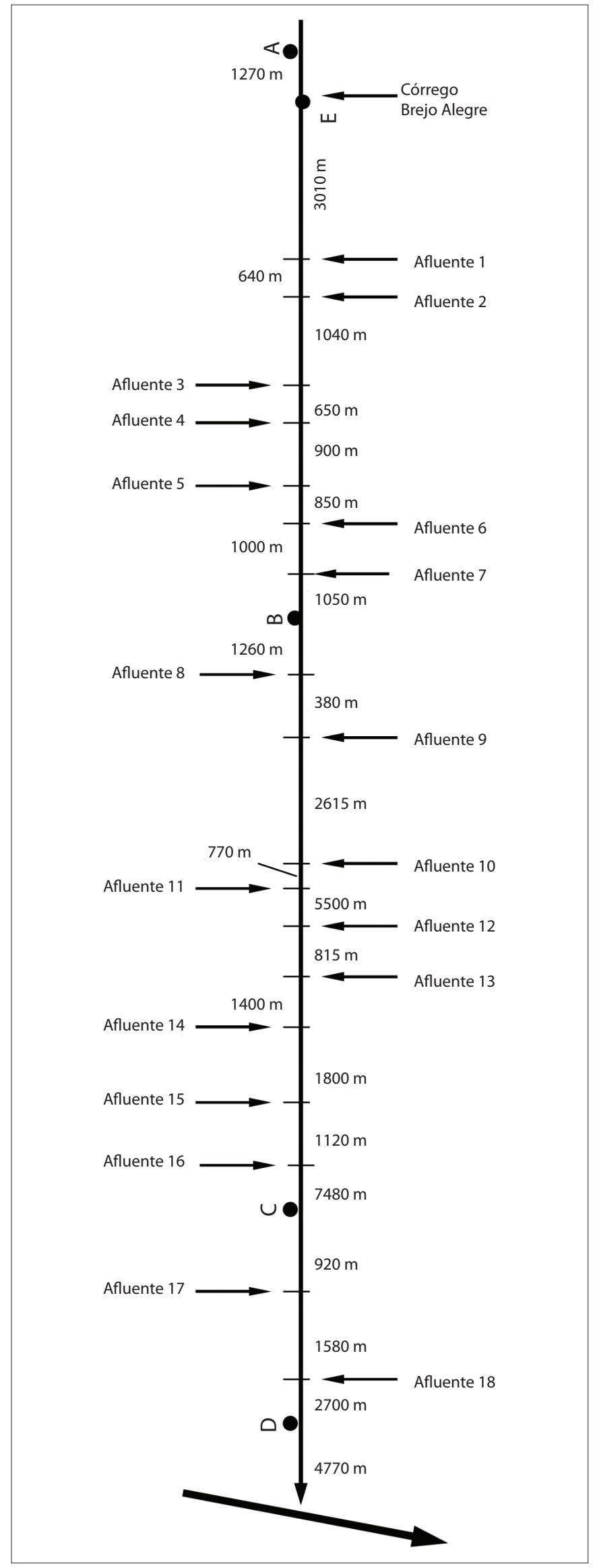

Figura 3 - Diagrama unifilar para o período de estiagem.
Solver do Excel, cujo critério de otimização é o melhor ajuste entre os parâmetros medidos e estimados. De acordo com a Equação 4, a otimização foi realizada por meio da maximização da função objetivo, definida pelo coeficiente de eficiência de Nash-Sutcliffe (KRAUSE; BOYLE; BÄSE, 2005):

NS $=1-\sum_{i}^{n}=1\left(X_{\text {med, } i}-X_{\text {est. }}\right)^{2} / \sum_{i}^{n}=1\left(X_{\text {med }, i}-\overline{X_{\text {med. }}}\right)^{2}$

onde, NS é o coeficiente de eficiência de Nash-Sutcliffe (NS igual a 1,0 indica ajuste perfeito), $X_{\text {med. }}$ é o valor do parâmetro medido, $X_{\text {est. }}$ é o valor do parâmetro estimado e $\overline{X_{\text {med. }}}$ é a média dos valores dos parâmetros medidos.

O coeficiente de reaeração $K_{2}$ foi calibrado a partir das constantes $\beta, \alpha$ e $\gamma$ da função $K_{2}=\beta$.v $\alpha \cdot h \gamma$, que resultou em $K_{2}\left(d i a^{-1}\right)=13,564$. $v^{0,758} \cdot h^{1,36}$, no qual $v$ é a velocidade média $\left(\mathrm{m} \cdot \mathrm{s}^{-1}\right)$ e $h$ é a profundidade média (m).

Com relação à concentração de $\mathrm{OD}$, na zona de mistura do córrego Brejo Alegre com o Rio Jordão existe um fraco decréscimo, prevalecendo a desoxigenação por demanda carbonácea e nitrificação sobre a reaeração natural do curso de água até aproximadamente $14.500 \mathrm{~m}$ a jusante do ponto A, no qual atinge o valor mínimo de $6.0 \mathrm{mgO}_{2} \cdot \mathrm{L}^{-1}$. A partir daí, o fenômeno da reaeração natural prevalece sobre a desoxigenação e alcança $6,7 \mathrm{mgO}_{2} \cdot \mathrm{L}^{-1}$ na sua foz no Rio Paranaíba. Os dados estimados de OD ajustaram-se bem àqueles medidos nos pontos B, C e D, resultando em um coeficiente de NashSutcliffe igual a 0,98.

$\mathrm{O}$ pico da $\mathrm{DBO}_{5}$ no Rio Jordão ocorreu logo na zona de mistura com o córrego Brejo Alegre, cujo valor foi de $8,3 \mathrm{mg}$. $\mathrm{L}^{-1}$. A partir da mistura, a DBO decresce gradualmente e atinge o valor de 6,0

Tabela 1 - Dados de entrada do efluente doméstico nos Cenários 1 e 2, além dos limites preconizados na Resolução Conama 430 (2011).

\begin{tabular}{|c|c|c|c|c|}
\hline \multicolumn{2}{|c|}{ Parâmetro* } & $\begin{array}{l}\text { Cená- } \\
\text { rio } 1\end{array}$ & $\begin{array}{l}\text { Cená- } \\
\text { rio } 2\end{array}$ & $\begin{array}{l}\text { CONAMA 430:2011 - } \\
\text { Classe } 2\left(\mathrm{em} \mathrm{mg} \cdot \mathrm{L}^{-1}\right)\end{array}$ \\
\hline \multirow{2}{*}{$\mathrm{DBO}_{5}$} & g.hab ${ }^{-1} \cdot$ dia $^{-1}$ & \multicolumn{2}{|c|}{54} & \multirow{2}{*}{$\begin{array}{c}\leq 120 \mathrm{mg} \mathrm{L}^{-1} \\
\geq 60 \% \text { remoção }\end{array}$} \\
\hline & \% remoção & 75 & 85 & \\
\hline OD & $\mathrm{mg} \cdot \mathrm{L}^{-1}$ & 0 & 1,5 & - \\
\hline \multirow{2}{*}{$\mathrm{N}_{\text {orgânico }}$} & g.hab-1 $\cdot$ dia $^{-1}$ & 5,0 & 5,0 & \multirow{2}{*}{ - } \\
\hline & \% remoção & 60 & 25 & \\
\hline \multirow{2}{*}{$\mathrm{NH}_{4}^{+}$} & g.hab-1 $\cdot$ dia $^{-1}$ & 7,0 & 7,0 & \multirow{2}{*}{$\leq 20 \mathrm{mg} \cdot \mathrm{L}^{-1} \mathrm{~N}$} \\
\hline & \% remoção & 50 & 85 & \\
\hline \multirow{2}{*}{$\mathrm{NO}_{2}^{-}$} & g.hab-1 $\cdot$ dia $^{-1}$ & 0 & 0 & \multirow{2}{*}{--} \\
\hline & \% remoção & 0 & 0 & \\
\hline \multirow{2}{*}{$\mathrm{NO}_{3}^{-}$} & g.hab-1 $\cdot$ dia $^{-1}$ & 0,5 & 0,5 & \multirow{2}{*}{--} \\
\hline & \% remoção & 60 & 25 & \\
\hline \multirow{2}{*}{$P_{\text {orgânico }}$} & g.hab-1 $\cdot$ dia $^{-1}$ & 1,0 & 1,0 & \multirow{4}{*}{--} \\
\hline & \% remoção & 35 & 25 & \\
\hline \multirow{2}{*}{$\begin{array}{l}P_{\text {inorgâ- }} \\
\text { nico }\end{array}$} & g.hab-1 $\cdot \mathrm{dia}^{-1}$ & 1,5 & 1,5 & \\
\hline & \% remoção & 35 & 25 & \\
\hline \multirow{2}{*}{ E-coli } & $\mathrm{NMP} / 100 \mathrm{~mL}$ & $10^{12}$ & $10^{12}$ & \multirow{2}{*}{---} \\
\hline & \% remoção & 90 & 60 & \\
\hline
\end{tabular}

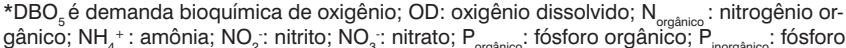
inorgânico; E-coli: Escherichia coli. 

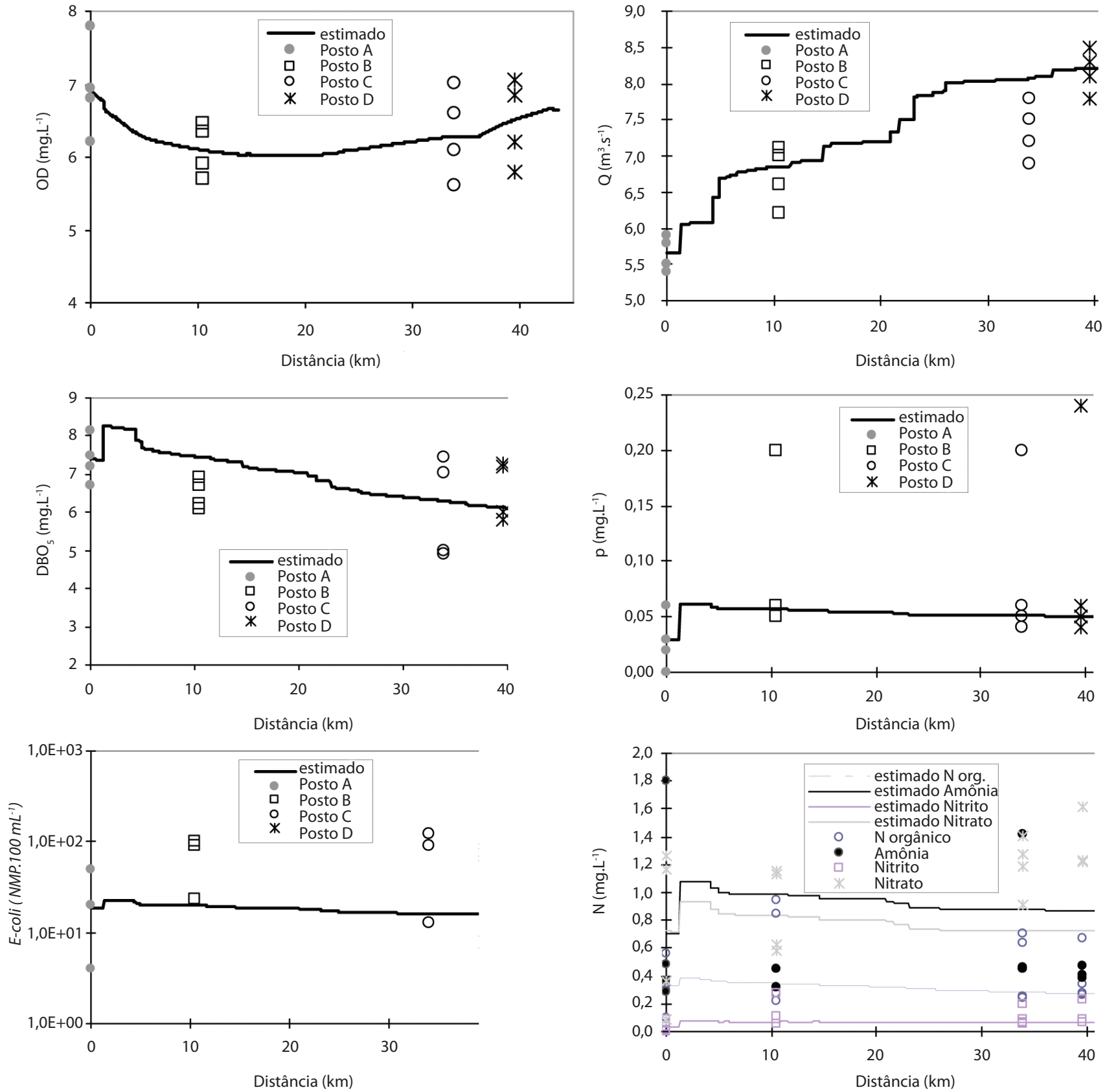

\begin{tabular}{|c|c|c|c|c|c|c|c|c|c|c|c|}
\hline Coeficiente & Valor & $\begin{array}{l}\text { Coeficiente } \\
\text { Tempera- } \\
\text { tura }\end{array}$ & Coeficiente & Valor & $\begin{array}{l}\text { Coeficiente } \\
\text { Tempera- } \\
\text { tura }\end{array}$ & $\begin{array}{l}\text { Coefi- } \\
\text { ciente }\end{array}$ & Valor & $\begin{array}{l}\text { Coeficiente } \\
\text { Tempera- } \\
\text { tura }\end{array}$ & Coeficiente & Valor & $\begin{array}{l}\text { Coeficiente } \\
\text { Tempera- } \\
\text { tura }\end{array}$ \\
\hline$K_{1}\left(\mathrm{dia}^{-1}\right)$ & 0,03 & 1,047 & $K_{\mathrm{spo}}\left(\mathrm{dia}^{-1}\right)$ & 0,001 & 1,024 & $K_{\mathrm{so}}\left(\mathrm{dia}^{-1}\right)$ & 0,001 & 1,024 & $\mathrm{O}_{2 \text { Namon }}\left(\mathrm{mgO}_{2} \cdot \mathrm{mgN}_{\text {amon }}{ }^{-1}\right)$ & 2,47 & - \\
\hline$K_{d}\left(\mathrm{dia}^{-1}\right)$ & 0,22 & 1,047 & $K_{\mathrm{oi}}\left(\mathrm{dia}^{-1}\right)$ & 0,40 & 1,047 & $K_{o a}\left(\operatorname{dia}^{-1}\right)$ & 0,40 & 1,047 & $\mathrm{O}_{2 \mathrm{Nnitri}}\left(\mathrm{mgO}_{2} \cdot \mathrm{mgN}_{\text {nitrito }}{ }^{-1}\right)$ & 0,00 & - \\
\hline$K_{\mathrm{s}}\left(\mathrm{dia}^{-1}\right)$ & 0,01 & 1,024 & $\begin{array}{l}S p_{\text {inorg }} \\
\left(\mathrm{g} \cdot \mathrm{m}^{-2} \cdot \mathrm{d}^{-1}\right)\end{array}$ & 0,10 & 1,074 & $K_{\text {an }}\left(\operatorname{dia}^{-1}\right)$ & 0,10 & 1,080 & $k_{\text {nitr }}$ & 0,53 & - \\
\hline$K_{2}\left(d i a^{-1}\right)$ & $\begin{array}{c}2,40- \\
6,42\end{array}$ & 1,024 & $K_{\mathrm{b}}\left(\mathrm{dia}^{-1}\right)$ & 0,15 & 1,07 & $\begin{array}{c}K_{\mathrm{nn}}\left(\mathrm{dia}^{-1}\right) \\
S_{\text {namon }} \\
\left(\mathrm{g} \cdot \mathrm{m}^{-2} \cdot \mathrm{d}^{-1}\right)\end{array}$ & $\begin{array}{l}1,0 \\
0,50\end{array}$ & 1,047 & $\mathrm{fNH}_{3}$ & $\begin{array}{r}7,00 \\
0,003\end{array}$ & - \\
\hline
\end{tabular}

Figura 4 - Parâmetros ao longo do trecho do Rio Jordão e coeficientes estimados e calibrados $\left(K_{1}\right.$ e $\left.K_{\mathrm{d}}\right)$. 
mg. $L^{-1}$ em sua foz no Rio Paranaíba. Apesar do coeficiente de NashSutcliffe igual a -0,28 evidenciar que não houve satisfatório ajuste entre dados estimados e medidos para o parâmetro DBO nos postos $\mathrm{B}, \mathrm{C}$ e D, observa-se que os intervalos de valores medidos e estimados ao longo do trecho do rio estudado mantiveram-se sempre no intervalo de 4,9 até 8,3 mg. $\mathrm{L}^{-1}$. São bastante conhecidas as implicações ecológicas resultantes das altas concentrações de nitrogênio presentes na água, principalmente aquelas na forma amoniacal, que estão ligadas à dinâmica do oxigênio no meio aquático. Na Figura 4 observou-se que as concentrações de nitrogênio e frações sempre apresentaram valores baixos, percebendo-se que os processos mais atuantes e responsáveis pela autodepuração do Rio Jordão no trecho estudado, para tal período de estiagem, foram apenas a reaeração natural e a demanda carbonácea. Os dados estimados superiores de nitrato e amônia em relação ao nitrogênio orgânico evidenciam a ocorrência da autodepuração por demanda nitrificada, baixa quando comparada à carbonácea.

Quanto ao parâmetro fósforo total, observa-se que, na confluência do córrego Brejo Alegre com o Rio Jordão, existe um acréscimo instantâneo da concentração, que alcança o valor de 0,062 mg. $\mathrm{L}^{-1}$, cuja redução é gradual até $0,051 \mathrm{mg} \cdot \mathrm{L}^{-1} \mathrm{em}$ sua foz no Rio Paranaíba (Figura 4). Observou-se que os parâmetros OD, nitrogênio e frações, fósforo total e E-coli respeitam os limites preconizados pela Resolução Conama 357:2005 em toda a sua extensão, de acordo com a classe 2, à qual o Rio Jordão enquadra-se. Com relação a $\mathrm{DBO}_{5}$, devido ao recebimento de carga poluidora do córrego Brejo Alegre, todo o trecho estudado encontra-se fora da regulamentação que estabelece o valor máximo de 5,0 mg. $\mathrm{L}^{-1}$.

\section{Simulações dos cenários}

Os coeficientes de reações e os dados qualitativos dos afluentes, do córrego Brejo Alegre e do Rio Jordão a montante da confluência (posto A) foram considerados semelhantes ao do período de estiagem (Tabela 2 e Figura 4). Os dados do efluente tratado e lançado na confluência foram estimados (Tabela 1).

Os resultados dos cenários são mostrados na Tabela 3, nos quais as simulações foram realizadas para a população do município em 2010 e para a máxima $P$ que os sistemas de tratamento suportariam para que, após lançado diretamente no Rio Jordão, a autodepuração natural do curso de água conseguisse adequar os parâmetros aos padrões da Resolução Conama 357:2005 para a Classe 2. Neste estudo, o parâmetro OD foi utilizado para estimar a população máxima e, posteriormente, avaliar o comportamento dos demais parâmetros.

De acordo com a Tabela 3, para a população de 2010 (109.800 habitantes), nos cenários 1 e 2, respectivamente, 3,4 e 9,2\% da extensão do trecho estudado entre a confluência do córrego Brejo Alegre com o Rio Jordão até sua foz respeita os limites da Resolução Conama 357:2005 com relação ao OD (mínimo de 5 mg. $\left.\mathrm{L}^{-1}\right)$. Com relação à DBO, todo o trecho encontra-se acima do limite máximo de 5mg.L ${ }^{-1}$ da Resolução Conama 357:2005 (7,410,9 mg. $\mathrm{L}^{-1}$ no Cenário 1 e 6,0-8,6 mg. $\mathrm{L}^{-1}$ no 2). Além disso, o nitrogênio e as frações mantiveram-se dentro das preconizações da legislação $\left(4,1-5,3 \mathrm{mg} . \mathrm{L}^{-1}\right.$ para o nitrogênio total no Cenário 1 e 3,9-5,0 mg.L $\mathrm{L}^{-1}$ para o nitrogênio total no 2). Quanto ao fósforo total $\left(0,74-0,97 \mathrm{mg} \cdot \mathrm{L}^{-1}\right.$ no Cenário 1 e 0,85-1,11 mg. $\mathrm{L}^{-1}$ no 2) e coliforme termotolerante E-coli $\left(1,1.10^{10}-1,6 \cdot 10^{10} \mathrm{NMP} .100\right.$ $\mathrm{mL}^{-1}$ no Cenário 1 e 4,4.10 ${ }^{10}-6,5 \cdot 10^{10} \mathrm{NMP} .100 \mathrm{~mL}^{-1}$ no 2), apenas $2,8 \%$ do trecho longitudinal está em acordo com a Resolução Conama 357:2005. Os desacordos observados para os parâmetros $\mathrm{OD}, \mathrm{DBO}_{5}$, fósforo total e coliforme termotolerante E-coli estão vinculados a uma vazão crítica no Rio Jordão 3,2 vezes inferior à menor medida no posto A em período de estiagem.

Para as simulações da estimativa de máxima população frente aos sistemas de tratamento de efluentes propostos, utilizando a concentração mínima de $5 \mathrm{mg}$. $\mathrm{L}^{-1}$ de OD como limitador, foi obtida a população de aproximadamente 13.300 e 20.500 habitantes, respectivamente, nos Cenários 1 e 2. Para estas, apenas os parâmetros fósforo total e E-coli mantiveram-se acima do limite máximo recomendado pela Resolução Conama 357:2005 em todo o trecho estudado (Tabela 3)

\section{Conclusões}

Nas simulações realizadas no período de estiagem, entre abril e julho de 2011, as calibrações dos parâmetros geraram resultados aceitáveis, principalmente para o ajuste dos dados simulados aos medidos de OD (coeficiente de Nash-Sutcliffe igual a 0,98), apesar da ausência de monitoramento de qualidade da água na maioria dos afluentes existentes no trecho do Rio Jordão estudado. Com o modelo

Tabela 3 - Resultados dos cenários simulados*, além dos limites preconizados na Resolução Conama 357:2005 para a Classe 2.

\begin{tabular}{|c|c|c|c|c|c|c|c|c|c|c|}
\hline Cenário & População & $\begin{array}{l}\text { Vazão } \\
\left(\mathrm{m}^{3} \cdot \mathrm{s}^{-1}\right)\end{array}$ & $\begin{array}{c}\mathrm{OD} \\
\left(\mathrm{mg} \cdot \mathrm{L}^{-1}\right)\end{array}$ & $\begin{array}{l}\mathrm{DBO}_{5} \\
\left(\mathrm{mg}^{-L^{-1}}\right)\end{array}$ & $\begin{array}{l}\mathrm{N}_{\text {orgânico }} \\
\left(\mathrm{mgg}^{-1} \mathrm{~L}^{-1}\right)\end{array}$ & $\begin{array}{c}\mathrm{NH}_{4}^{+} \\
\left(\mathrm{mg} \cdot \mathrm{L}^{-1}\right)\end{array}$ & $\begin{array}{c}\mathrm{NO}_{2}^{-} \\
\left(\mathrm{mg} \cdot \mathrm{L}^{-1}\right)\end{array}$ & $\begin{array}{c}\mathrm{NO}_{3}^{-} \\
\left(\mathrm{mg} \cdot \mathrm{L}^{-1}\right)\end{array}$ & $\begin{array}{c}\mathrm{P}_{\text {total }} \\
\text { (mg.L-1) }\end{array}$ & $\begin{array}{c}E \text {-coli } \\
\text { (NPM.100 } \mathrm{mL}^{-1} \text { ) }\end{array}$ \\
\hline \multirow{2}{*}{1} & 109800 & $2,25-2,94$ & $1,3-5,5$ & $7,4-10,9$ & $0,82-1,45$ & $2,44-2,86$ & $0,06-0,12$ & $0,72-0,88$ & $0,74-0,97$ & $1,1.10^{10}-1,6.10^{10}$ \\
\hline & 13300 & $1,92-2,62$ & $5,0-6,6$ & $3,0-4,1$ & $0,29-0,52$ & $1,0-1,3$ & $0,07-0,09$ & $0,74-0,91$ & $0,14-0,18$ & $1,4.10^{9}-2,3.10^{9}$ \\
\hline \multirow{2}{*}{2} & 109800 & $2,25-2,94$ & $2,6-5,8$ & $6,0-8,6$ & $1,35-2,44$ & $1,47-1,63$ & $0,06-0,09$ & $0,80-0,98$ & $0,85-1,11$ & $4,4.10^{10}-6,5.10^{10}$ \\
\hline & 20500 & $1,95-2,64$ & $5,0-6,6$ & $2,9-3,8$ & $0,43-0,81$ & $1,01-1,14$ & $0,07-0,08$ & $0,75-0,93$ & $0,21-0,28$ & $8,6 \cdot 10^{9}-1,4 \cdot 10^{10}$ \\
\hline \multicolumn{2}{|c|}{ CONAMA 357} & - & $\geq 5,0$ & $\leq 5,0$ & - & 3,70 & 1,0 & 10,0 & 0,1 & $10^{3}$ \\
\hline
\end{tabular}

*mínimo-máximo; $\mathrm{OD}$ : oxigênio dissolvido; $\mathrm{DBO}_{5}$ : demanda bioquímica de oxigênio; $\mathrm{N}_{\text {orgânico: }}$ : nitrogênio orgânico; $\mathrm{NH}_{4}^{+}:$amônia; $\mathrm{NO}_{2}:$ nitrito; $\mathrm{NO}_{3}:$ nitrato; $\mathrm{P}_{\text {total }}$ : fósforo total; E-coli: Escherichia coli. 
calibrado, os cenários simulados para a população de 2010 mostraram que o Rio Jordão não possui capacidade de autodepuração natural para efluente tratado pelo sistema 'australiano' e lodo ativado convencional, principalmente para uma análise sobre os parâmetros 'OD' e 'DBO'. Apenas 12,1\% da população de 2010 no Cenário 1 e 18,7\% no 2 é que fariam com que a concentração de OD ficasse acima do limite mínimo da Resolução Conama 357:2005. Este estudo forneceu subsídios para atentar sobre o problema de lançamento de despejos acima do que possa suportar o Rio Jordão e, também, alertou para a importância do monitoramento constante de quantidade e qualidade da água em cursos de água como subsídio para modelos confiáveis e melhor gerenciamento dos recursos hídricos.

\section{Referências}

ALEM SOBRINHO, P. (2001) Tratamento de esgoto e produção de lodo. In: Biossólidos na agricultura. São Paulo: Sabesp, p. 7-40.

AMORIM JUNIOR, J.C.; LIMA, L.C.S.; VIEIRA, P.L.C. (2011) Estudo da autodepuração em trecho do rio Jucu, Vila Velha - ES, utilizando os modelos matemáticos QUAL-UFMG e Streeter-Phelps. In: $26^{\circ}$ Congresso Brasileiro de Engenharia Sanitária e Ambiental, ABES, Porto Alegre, Rio Grande do Sul.

ARNOLD, U.; ORLOB, G.T. (1989) Decision support for estuarine water quality management. Journal of Water Resources Planning and Management, v. 115, n. 6, p. 775-792.

ABNT - Associação Brasileira de Normas Técnicas. (1986) Projeto de redes coletoras de esgoto sanitário, NBR 9649. Rio de Janeiro.

ABNT - Associação Brasileira de Normas Técnicas. (1992) Projeto de estações de tratamento de esgoto sanitário, NBR 12209. Rio de Janeiro.

ABNT - Associação Brasileira de Normas Técnicas. (2000) Sistemas enterrados para condução de esgoto sanitário - Projeto de redes coletoras com tubos de PVC, NBR 14486. Rio de Janeiro.

BRASIL. (2005) Conselho Nacional do Meio Ambiente. Resolução nº 357, Diário Oficial da União, de 18 de março de 2005.

BRASIL. (2011) Conselho Nacional do Meio Ambiente. Resolução n 430, Diário Oficial da União, de 16 de maio de 2011.

BRASIL. (2010) Instituto Brasileiro de Geografia e Estatística - IBGE. Censos demográficos. Disponível em: <http://www.ibge.gov.br/ cidadesat/topwindow.htm> . Acesso em: 09 jan. 2012.

CHAPRA, S.C. (1997) Surface water quality modeling. New York: McGraw-Hill, 844 p.

CHAPRA, S.C.; PELLETIER, G.J. (2006) QUAL2K: a Modeling Framework for simulating river and stream water quality: documentation and user manual. Civil and environmental Engineering Department, Tufts University, Medford, MA, $109 \mathrm{p}$.

DA SILVA, T.C.; SOUZA, L.L.; CAIADO, M.A.C.; REIS, J.A.T. (2011) Avaliação da capacidade de assimilação de compostos de nitrogênio em rios a partir da aplicação de modelo matemático de qualidade de água. In: $26^{\circ}$ Congresso Brasileiro de Engenharia Sanitária e Ambiental, $A B E S$, Porto Alegre, Rio Grande do Sul.
DE PAULA, L.M. (2011) Avaliação da qualidade da água e autodepuração do rio Jordão, Araguari (MG). Dissertação de Mestrado, Universidade Federal de Uberlândia, Engenharia Civil, Uberlândia, 177 p.

GUEDES, H.A.S. (2009) Modelagem da qualidade da água e da capacidade de autodepuração do rio Pomba. Dissertação de Mestrado, Universidade Federal de Viçosa, Engenharia Agrícola, Viçosa, 149 p.

IDE, W.R. \& RIBEIRO, M.L. (2009) Calibração do modelo de qualidade da água QUAL-UFMG para o rio Taquarizinho em período de estiagem. Relatório de Iniciação Científica CNPq, Engenharia Ambiental, Universidade Federal de Mato Grosso do Sul.

KRAUSE, P.; BOYLE, D.P.; BÄSE, F. (2005) Comparison of different efficiency criteria for hydrological model assessment. Advances in Geosciences, v. 5, p. 89-97.

PAREDES, J. \& LUND, J.R. (2006) Refill and drawdown rules for paralle reservoirs: quantity and quality. Water Resources Management, v. 20, n. 1, p. 359-376.

PAREDES, J.; ANDREU, J.; SOLERA, A. (2010) A decision support system for water quality issues in the Manzanares River (Madrid, Spain). Science of the Total Environment, v. 408, n. 12, p. 2576-2589.

PEREIRA, L.H.; MIRANDA, C.S.; TEODORO, A.; RIBEIRO, M.L. (2011) Estudo da capacidade de autodepuração no córrego Lagoa. In: $26^{\circ}$ Congresso Brasileiro de Engenharia Sanitária e Ambiental, ABES, Porto Alegre, Rio Grande do Sul.

REICHERT, P. (1998) AQUASIM 2.0 - Tutorial. Swiss Federal Institute for Environmental Science and Technology (EAWAG).

STRZEPEK, K.; GARCÍA, L.; OVER, T. (1989) MITSIM 2.1 river basin simulation model, user manual. Center for Advanced Decision Support for Water and Environmental Systems, University of Colorado, Boulder.

TCHOBANOGLOUS, G.; BURTON, F.L.; STENSEL, H.D. (2003) Wastewater Engineering: Treatment and reuse. 4. ed. Boston: McGraw-Hill, 1848 p.

UNIVERSIDADE FEDERAL DE VIÇOSA. (2009) Hidrotec. Disponível em: <http://www.hidrotec.ufv.br>. Acesso em: 10 jan. 2012

VON SPERLING, M. (2007) Estudos e modelagem da qualidade da água de rios. Departamento de Engenharia Sanitária e Ambiental, Universidade Federal de Minas Gerais, Belo Horizonte, UFMG, v. 7, 588 p. 